\title{
Method of pectin esterification determination degree by titrated acidity
}

\author{
Oksana Shulga, Volodymyr Lystopad, \\ Sergii Shulga, Lyudmila Yurchuk
}

\section{National University of Food Technologies, Kyiv, Ukraine}

Keywords:

Pectin

Esterification

Acidity

IR-spectroscopy

NMR-spectrums

Article history:

Received 19.09.2019

Received in revised

form 11.12.2019

Accepted 30.06.2020

Corresponding

author:

Oksana Shulga

E-mail:

shulgaos@ukr.net

DOI: $10.24263 / 2304-$

974X-2020-9-2-10

\section{Abstract}

Introduction. Studies have been conducted to develop a method for determining the pectin esterification degree in order to limit the use a significant number of costly and hardly available reagents.

Materials and methods. The samples of apple and citric pectin were exhibited at Kyiv market with the different degree of esterification according to accompanying documentation. IR-spectroscopy research was provided on device Nexus-475 Nicolet firm. NMR-spectrums were registered by NMRspectrometer Mercury, VARIAN firm. Mathematical treatment of the results is done according to mathematical modeling concept.

Results and discussion. In the IR spectrum of lowesterified pectin, this band is low intensity and is in the oscillations region of $1686.71 \mathrm{~cm}^{-1}$. In spectrum of high-ester pectin the intensive line with three maximums at 3400.56 $\mathrm{cm}^{-1}, 3316.52 \mathrm{~cm}^{-1}, 3271.70 \mathrm{~cm}^{-1}$, which corresponds to stretching $\mathrm{vOH}$. In IR-spectrums with esterification till $42 \%$ the line of free carboxyl group is available, in IR-spectrums of high-esterified pectin there is an intensive line of carboxylate groups $\left(\mathrm{CO}^{2-}\right)$, and that differs the given spectrums.

The given characteristics of NMR-spectrums show the difference in structure of high- and low-esterified pectin, but it does not give an opportunity to conduct the quantitative determination of esterification degree.

While analyzing the results we can make a conclusion that the degree or hyperbolic models are the best for prognosing.

The difference between chemical method of esterification degree determination and suggested method is $0.6-1.3 \%$.

Conclusions. Determination of the pectin degree esterification is possible by tyranic acidity with subsequent calculation by the regression equation. 


\section{Introduction}

One the most important nutritional and technological characteristics of pectin is its esterification degree [1]. The determining of this indicator needs the use of essential amount of reagents according to approved methods and time. A task was set up to find an alternative method of determining the degree of pectin esterification, since it is necessary to know the degree of pectin esterification in order to produce marmalade products.

According to the known method by national standard of Ukraine the esterification degree is a correlation of polygalacturonic acid esterified carboxyl groups to their general amount in pectin. This method of determination of pectin esterification degree needs the following reagents: hydrochloric acid, sodium hydroxide, ethyl alcohol rectified, silver nitrate, indicator alizarin, ammonia aqueous solution concentrate, bromothymol blue, cresol red, phenol red.

In literature there are data about the alternative methods of determination of pectin esterification degree such as the suggested method with the use IR-spectroscopy [2, 3, 7], NMR-spectroscopy [8], chromatography [9]. It is developed the improved method of highperformance liquid chromatography (HPLC) for simultaneous determination of pectin degrees of methylation and acetylation [5]. The suggested way includes the saponification in heterogeneous environment with the next separation of methanol, acetic acid and inner standard on C18 column and the quantitative determination with the help of refractometry [6]. But the suggested alternative methods need the use of special expensive equipment: IRand NMR-spectroscopes and chromatograph $[4,8]$. That is why the search of the alternative method which does not need the use of expensive equipment and big amount of reagents on determination of pectin esterification degree remains actual.

The purpose of the study is to develop a method for determining the esterification degree using a minimum number of reagents, which does not require a significant amount of time.

\section{Materials and methods}

\section{Materials}

Samples of apple and citrus pectin presented with different esterification degrees.

\section{Methods}

The total acidity was determined by the titration method of pectin batch solution in the presence of phenolphthalein [16].

The esterification degree was determined method that is based on the titrimetric analysis of free and after saponification esterified carboxyl groups of polygalacturonic acid in pectin batch, which is purified from soluble ballast additives and cations [16].

The presence of a carboxyl group in pectin of different esterification degrees was determined by IR spectroscopy [7]. IR-spectroscopy research was provided on device Nexus475 Nicolet firm, in pills with $\mathrm{KBr}$.

The chemical shift of the protons that are part of the pectin structural components was recorded using NMR spectra [8]. NMR-spectrums were registered by NMR-spectrometer Mercury, VARIAN firm, $400 \mathrm{MHz}$ in the solution DMSO- $\mathrm{d}_{6}$. 


\section{Food Technology}

\section{Processing of research results}

Mathematical treatment of the results is done according to mathematical modeling concept [11].

\section{Results and discussion}

With the aim to get the complete characteristics of pectin properties with different degree of esterification, except total acidity, IR- and NMR-researches of chosen pectin samples were conducted.

The structure of pectin with different degrees of esterification is characterized by the presence of methylated carboxyl groups (see Figure 1).
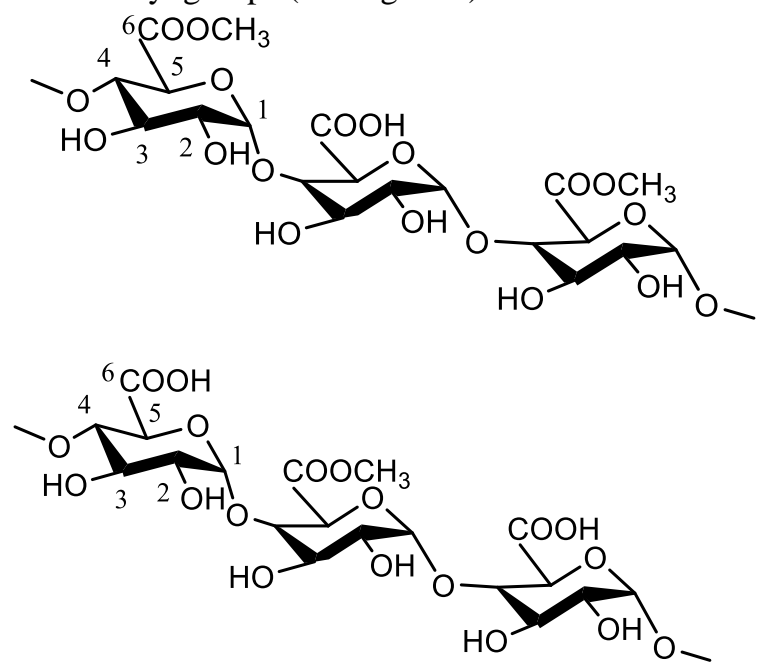

a

b

Figure. 1. Pectin structure: a - highly esterified; $b$ - low esterified

The given different pectin structure is confirmed by IR- and NMR-spectrometric researches, which are given further.

\section{IR-spectroscopy research}

According to the above given literature data [12], high- and low-esterified pectin have different IR-spectrums.

The IR-spectrums of high-ester (58-62\%) and low-ester pectin (27-33\%) Figure 2. 

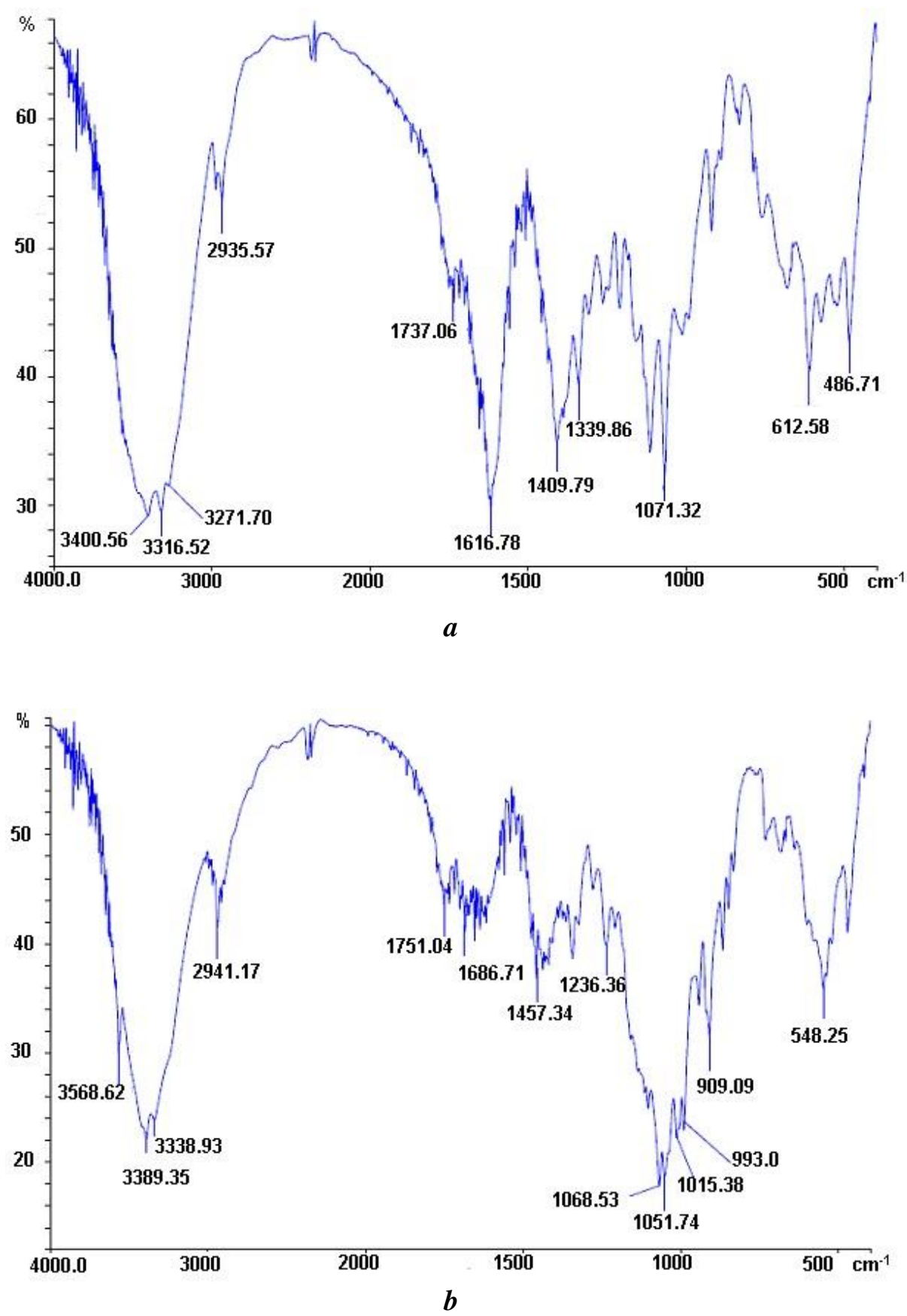

Figure 2. IR-spectrums of pectin: a - high-ester; $b$ - low-ester 


\section{— Food Technology —}

In spectrum of high-ester pectin the intensive line with three maximums at 3400.56 $\mathrm{cm}^{-1}, 3316.52 \mathrm{~cm}^{-1}, 3271.70 \mathrm{~cm}^{-1}$ is presented, which corresponds to stretching $\mathrm{vOH}$. In IRspectrum of low-ester pectin this line has maximum at $3568.62 \mathrm{~cm}^{-1}$, separated from the other two $3389.35 \mathrm{~cm}^{-1}$ is located in weaker vibrations region, that shows a greater degree of hydrogen bonds, and confirms a lower degree of esterification. Maximum at $3568.62 \mathrm{~cm}^{-}$ ${ }^{1}$ it corresponds to stretching of free carboxyl group, that also confirms low esterification degree.

The line at $2935.57 \mathrm{~cm}^{-1}$ of high-ester pectin sample and at $2941.41 \mathrm{~cm}^{-1}$ of low-ester sample are due to the presence of asymmetric and symmetric vibrations $v \mathrm{C}-\mathrm{H}$, which are located in remainders of galactopyranose rings of pectin.

In both spectrums of low- and high-ester pectin samples lines at $1737.06 \mathrm{~cm}^{-1}$ and at $1751.04 \mathrm{~cm}^{-1}$ of weak intensity exist, which correspond to vibrations of ester group $\mathrm{C}=\mathrm{O}$ in composition of high- and low-ester pectin respectively.

In IR-spectrum of high-ester pectin sample the presented line of high intensity at 1616.78 $\mathrm{cm}^{-1}$ belongs to latitudinal vibrations of adsorbed related water that is crossed by asymmetric vibrations of carboxylate ion $\left(\mathrm{CO}^{2-}\right)$. In IR-spectrum of low-ester pectin sample this line is of weak intensity and is located in vibration region at $1686.71 \mathrm{~cm}^{-1}$.

Besides this, IR-spectrums samples with esterification degree 24\%, 28-36\%, 38\%, 36$42 \%, 65-68 \%, 66-68 \%$ were received. In IR-spectrums with esterification till $42 \%$ the line of free carboxyl group is available, in IR-spectrums of high-ester pectin there is an intensive line of carboxylate groups $\left(\mathrm{CO}^{2-}\right)$, and that differs the given spectrums.

\section{NMR-researches research}

NMR-spectrums of high- and low-ester pectin samples shown on Figure 3.

D-galacturonic acid in pectin is in conformational form "chair". Meanwhile, the hydroxyl groups near Carbon atom 1 and 4 are in axial position, so free rotation around glycoside bond is complicated and pectin acid can be seen as a chain with limited flexibility, that changes the electron density a little around galactopyranose ring protons. The higher density is, the greater influence on inner field is, and therefore in a stronger field the resonance signal of according proton will appear.

The influence of this factor corresponds that the proton, which has more acidic properties (with less density of electronic shell) resonates in weaker field [12].

In general case the position of proton signal depends on electron density at surrounding it atoms, which are determined in such case by inductive and resonance effects, that are transmitted by chemical bonds and anisotropic effect of non-connected atom (interaction "through the space") [13].

Taking into consideration the above given and literature data [14] in NMR-spectrums of high- and low-ester pectin samples signals are interpreted as shown in Table 1. 

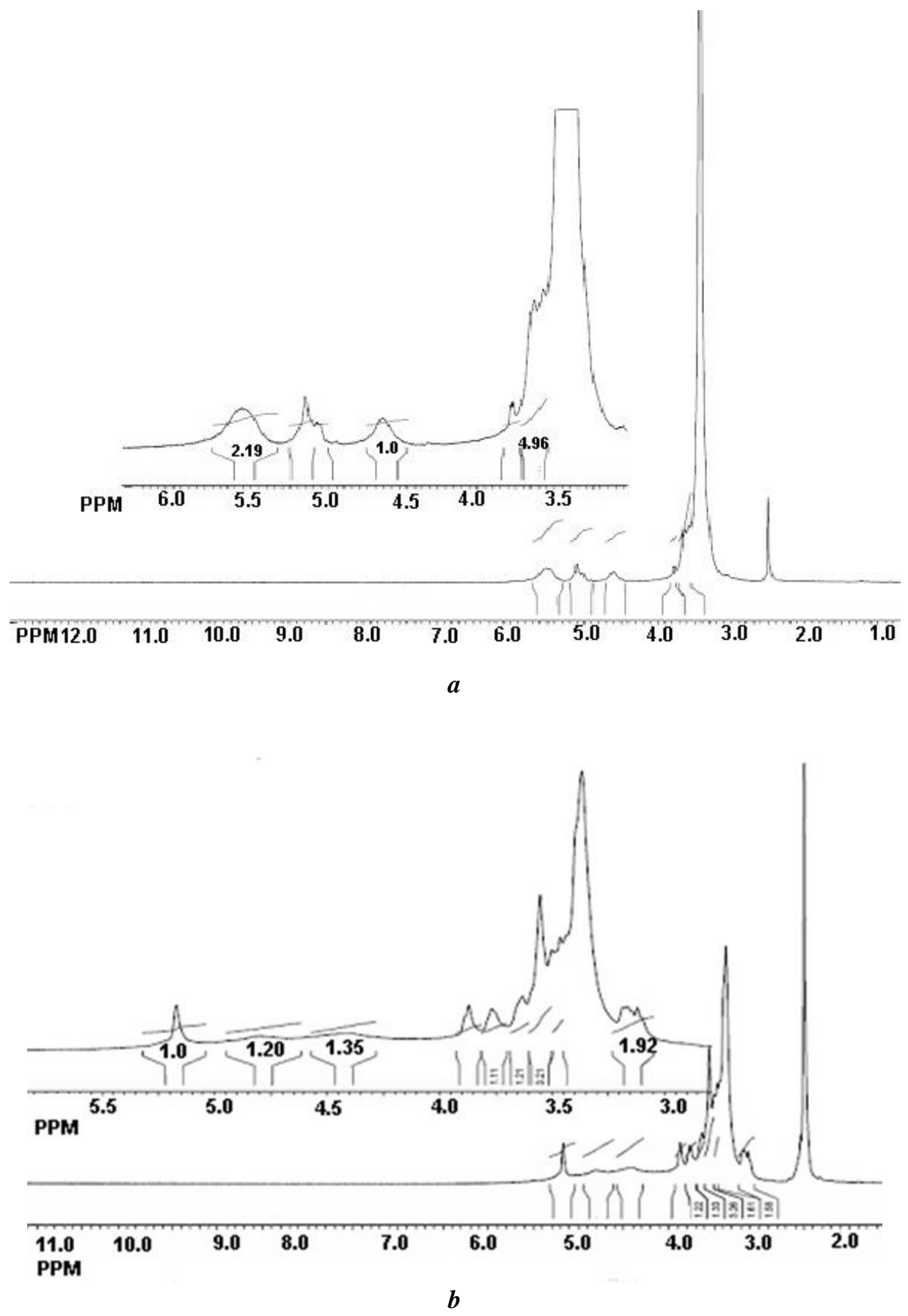

Figure 3. NMR-spectrums of pectin: $\boldsymbol{a}$ - high-ester; $\boldsymbol{b}$ - low-ester 
Table 1

Interpretation of NMR-spectrums of high- and low-ester pectin

\begin{tabular}{|c|c|c|}
\hline \multirow{2}{*}{ Proton type } & \multicolumn{2}{|c|}{ Value of chemical shift ppm } \\
\cline { 2 - 3 } & High-ester pectin & Low-ester pectin \\
\hline$-\mathrm{OCH}_{3}$ & 3.579 & 3.567 \\
\hline $4-\mathrm{H}$ & 3.632 & 3.639 \\
\hline $5-\mathrm{H}$ & $3.780^{*}$ & 3.769 \\
\hline $3-\mathrm{H}$ & 4.605 & 3.870 \\
\hline $2-\mathrm{OH}$ & 5.102 & 4.425 \\
\hline $1-\mathrm{H}$ & 5.514 & 4.805 \\
\hline $3-\mathrm{OH}$ & - & 5.170 \\
\hline $2-\mathrm{H}$ & - & 3.184 \\
\hline
\end{tabular}

*signals overlapped one by one

The given characteristics of NMR-spectrums shows the difference in structure of highand low-ester pectin, but it does not give an opportunity to conduct the quantitative determination of esterification degree.

\section{Total pectin acidity research}

Results of research of total acidity determination of pectin samples shown on Figure 4.

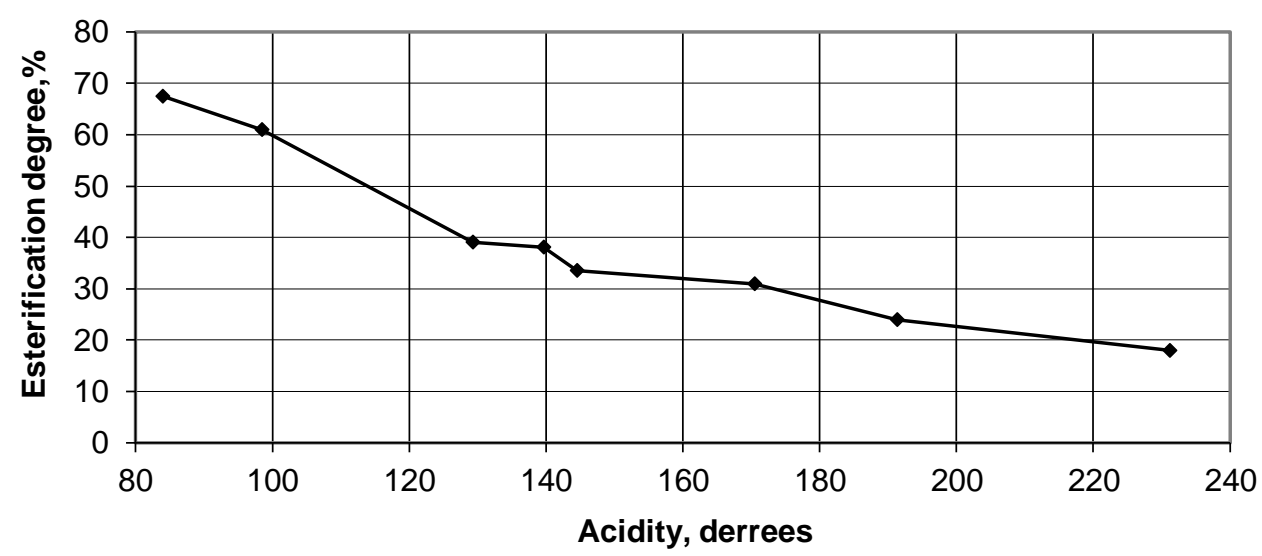

Figure 4. Total acidity change depending on pectin esterification degree

According to the given results (see Figure 4) there is a certain dependence between total acidity and degree of pectin esterification. It gives the base for searching the mathematical model. 


\section{- Food Technology}

\section{Mathematical processing of research results}

On the base of the received experimental data the mathematical treatment will be conducted.

According to the dependence graph outlook Y $(\%)$ esterification degree from X (degr.) acidity and big tightness of linear connection $\left(r_{y x} \approx-0,96\right)$, we will look through the linear dependence. In order to clarify the best outlook of the resultative factor approximation we will use other kinds of regression equation: hyperbolic, exponential and degree dependence:

$$
\begin{gathered}
\hat{y}=a_{0}+a_{1} x_{1}-\operatorname{linear}(1) \\
\hat{y}=a_{0}+\frac{a_{1}}{x},- \text { hyperbolic }(2) \\
\hat{y}=a_{0} e^{a_{1} x}-\text { exponential }(3) \\
\hat{y}=a_{0} x^{a_{1}}-\text { degree }(4)
\end{gathered}
$$

where $a_{0}, a_{1}$ - are unknown regression dependence coefficients.

The unknown model parameters (1-4) $\left(a_{0}, a_{1}\right)$ we will find by the least squares method. For the formation of methods of receiving the unknown models parameters (1-4) we will consider them as common dependence $y(x)$.

For the model (1) we will use function LINEAR from Microsoft Excel (category STATISTICAL), which also contains additional characteristics for the model qualitative analysis.

The model of inverted connection with the help of substitute $t_{i}=\frac{1}{x_{i}}$ is reduced to linear $y=a_{o}+a_{1} t$.

To build the exponential model it is necessary to take logarithm of left and right parts (3). We will get $\ln y=\ln a_{o}+a_{1} x$, so as use the function LINEAR for data $\left(\ln y_{i} ; x_{i}\right)$.

For the degree function after taking logarithm as in previous case we will get $\ln y=\ln a_{o}+a_{1} \ln x$ and use function LINEAR for data $\left(\ln y_{i} ; \ln x_{i}\right)$. The inverted formulae for finding $a_{o}=e^{\ln a_{o}}$ based on basic logarithmic identity.

Comparison quantitative and qualitative characteristics of received models and choosing of "the best" for forecast carried out with the help of additional regression statistics.

1. Coefficient (index) of determination $R^{2}$, is a part of dispersion, which explains regression (model criterion of adequacy, which is the measure of independent variable $\mathrm{x}$ explanatory force $x$ );

2. F - Fisher statistics, univocally gives an answer about model adequacy (an opportunity of use on practice);

3. Sum of squares of remainders (errors) $S S E=\sum_{i=1}^{n}\left(y_{i}-y_{i}\right)^{2}$ (deviation square of real values of independent variable $y_{i}$, from calculated $y_{i}$ ); 


\section{— Food Technology}

4. $M A P E=\frac{1}{n} \sum_{i=1}^{n} \frac{\left|y_{i}-y_{i}\right|}{y_{i}} 100 \%$ - absolute average percent error (indicator of prognosis immutability). This criterion is used while comparing prognosis exactness, because it characterizes the related prognosis exactness.

Meanwhile it is considered that that the definition $M A P E$ is less than $10 \%$. It gives high prognosis exactness and the model quality.

These quality criteria are used as additional information while choosing the best model from possible ones.

In calculating the additional models quantities (1-4) we will use formulae with [15].

$$
\begin{aligned}
& S S R=\sum_{i=1}^{n}\left(y_{i}-\bar{y}\right)^{2}-\text { sum of squares, that explains the regression; } \\
& S S T=\sum_{i=1}^{n}\left(y_{i}-\bar{y}\right)^{2}-\text { total sum of deviation squares; } \\
& R^{2}=\frac{S S R}{S S T} .
\end{aligned}
$$

For the linear model according parameters the egality $S S T=S S R+S S E$ is done.

While verifying the model adequacy we will use F-test criterion.

To that end we find the calculated criterion definition

$$
F_{\text {estimated }}=\frac{S S R /(m-1)}{S S E /(n-m)}=\frac{R^{2} /(m-1)}{\left(1-R^{2}\right) /(n-m)},
$$

where $m$ - quantity of the unknown parameters in the model $(m=2)$,

$\mathrm{n}$ - quantity of data $(n=7)$.

For the assigned level of significance (error) $\alpha$ and number of freedom degrees $m-1$ and $n-m$ we find from the statistical tables (or in Microsoft Excel function FINV) $F_{c r}$. If $F_{\text {calc }}>F_{c r}$, the model is adequate, otherwise no.

To verify the significance of the received equations coefficients we will use t-test criterion. To that end we will calculate the calculated definitions of the criterion on the formulae $t_{\text {icalc }}=\left|\mathrm{a}_{\mathrm{i}}\right| / \sigma_{\mathrm{i}}, i=0.1$, and will compare it with the table one for $5 \%$ level of significance. While using t-test we calculate according to the number of freedom degrees $n-m=5$ and level of significance $\alpha=0.05$, we will get $t_{c r}+2.57$. The dispersions of models parameters deviations we will calculate on the formulae:

$$
\sigma_{o}^{2}=\sigma_{\varepsilon}^{2} \cdot \frac{\sum_{i=1}^{n} x_{i}^{2}}{n \cdot \sum_{i=1}^{n}\left(x_{i}-\bar{x}\right)^{2}}, \sigma_{1}^{2}=\frac{\sigma_{\varepsilon}^{2}}{\sum_{i=1}^{n}\left(x_{i}-\bar{x}\right)^{2}}, \sigma_{\varepsilon}^{2}=\frac{S S E}{n-m} .
$$

All the received results we will put into the Table 2. 
Results of dispersions calculation of parameters deviations of the suggested models

\begin{tabular}{|l|c|c|c|c|c|}
\hline \multirow{2}{*}{ Model outlook } & \multicolumn{5}{|c|}{ Parameters of t-criterion } \\
\cline { 2 - 6 } & $\sigma_{o}$ & $\sigma_{1}$ & $t_{\text {calc }}$ & $t_{\text {lcalc }}$ & $t_{\text {cr }}$ \\
\hline Linear & 7.68 & 0.05 & 12.78 & 7.54 & \pm 2.57 \\
\hline Inverted & 3.51 & 430.05 & 3 & 15.56 & \pm 2.57 \\
\hline Exponential & 4.68 & 0.0008 & 31.48 & 11.84 & \pm 2.57 \\
\hline Degree & 0.028 & 3.947 & 4553.85 & 44.81 & \pm 2.57 \\
\hline
\end{tabular}

Through the bilateral Student's t-test, it is easy to make sure, using Table 3, that the parameters of all the received models are significant at $5 \%$ level.

Results of models significance verification at $5 \%$ level

Table 3

\begin{tabular}{|l|l|c|c|c|c|}
\hline \multicolumn{1}{|c|}{ Model type } & \multicolumn{1}{|c|}{ Equations } & $\mathbf{R}^{2}$ & $\begin{array}{c}\text { F-test } \\
\text { criterion }\end{array}$ & SSE & MAPE, \% \\
\hline Linear & $\hat{y}=98-0.41 x$ & 0.92 & 56.85 & 125.59 & 11 \\
\hline Hyperbolic & $\hat{y}=-10.55+\frac{6691.08}{x}$ & 0.98 & 242.08 & 31.44 & 5 \\
\hline Exponential & $\hat{y}=147.3 \cdot e^{-0.01 x}$ & 0.98 & 145.63 & 46.65 & 6 \\
\hline Degree & $\hat{y}=17974.9 \cdot x^{-1.25}$ & 0.996 & 242.08 & 33.20 & 5 \\
\hline
\end{tabular}

While analyzing the results of Table 3 we can make a conclusion that the degree or hyperbolic models are the best for prognosing.

$$
\begin{gathered}
\hat{y}=17974.9 \cdot x^{-1.25} \\
\hat{y}=-10.55+\frac{6691.08}{x}
\end{gathered}
$$

Let us verify the received models. On condition of the well-known value of pectin titrated acidity 139.7 degr., the degree of esterification is 36.9 and $37.4 \%$, for the first and second models consequently, while by the chemical method it was $38 \%$. On condition of the well-known value of pectin titrated acidity $191.3 \mathrm{degr}$., the calculated value esterification degree is $29.9 \%$ and $24.4 \%$, by the chemical method it was $24.0 \%$.

In this way, the found models are adequate and available for use.

\section{Conclusions}

1. High-ester and low-ester pectin characteristics with the help of IR- and NMRspectroscopy is held, whereas these methods do not give the univocal indication of esterification degree.

2. The pectin titrated acidity determination is held with the mathematical treatment. As a result, two regression equations are received.

3. The difference between chemical method of esterification degree determination and suggested method is $0.6-1.3 \%$. 


\section{Food Technology}

\section{References}

1. Gnanasambandam R., Proctor A. (2000), Determination of pectin degree of esterification by diffuse reflectance Fourier transform infrared spectroscopy. Food chemistry, 68(3), pp. 327-332.

2. Chatjigakis A. K., Pappas, C., Proxenia N., Kalantzi O., Rodis, P., \& Polissiou M. (1998), FT-IR spectroscopic determination of the degree of esterification of cell wall pectins from stored peaches and correlation to textural changes. Carbohydrate Polymers, 37(4), pp. 395-408.

3. Manrique G. D., \& Lajolo F. M. (2002), FT-IR spectroscopy as a tool for measuring degree of methyl esterification in pectins isolated from ripening papaya fruit. Postharvest Biology and Technology, 25(1), pp. 99-107.

4. Monsoor M. A., Kalapathy U., \& Proctor A. (2001), Improved method for determination of pectin degree of esterification by diffuse reflectance Fourier transform infrared spectroscopy. Journal of Agricultural and Food Chemistry, 49(6), pp. 27562760. DOI: $10.1021 /$ jf0009448

5. Fellah A., Anjukandi P., Waterland M. R., \& Williams M. A. (2009), Determining the degree of methylesterification of pectin by ATR/FT-IR: Methodology optimisation and comparison with theoretical calculations. Carbohydrate polymers, 78(4), pp. 847-853.

6. Pappas C. S., Malovikova A., Hromadkova Z., Tarantilis P. A., Ebringerova A., \& Polissiou M. G. (2004), Determination of the degree of esterification of pectinates with decyl and benzyl ester groups by diffuse reflectance infrared Fourier transform spectroscopy (DRIFTS) and curve-fitting deconvolution method. Carbohydrate Polymers, 56(4), pp. 465-469.

7. Barros A. S., Mafra I., Ferreira D., Cardoso S., Reis A., da Silva J. L., \& Coimbra M. A. (2002), Determination of the degree of methylesterification of pectic polysaccharides by FT-IR using an outer product PLS1 regression. Carbohydrate Polymers, 50(1), pp. 85-94.

8. Grasdalen H., Bakøy, O. E., \& Larsen B. (1988), Determination of the degree of esterification and the distribution of methylated and free carboxyl groups in pectins by 1H-NMR spectroscopy. Carbohydrate Research, 184, pp. 183-191.

9. Voragen A. G. J., Schols H. A., \& Pilnik W. (1986), Determination of the degree of methylation and acetylation of pectins by HPLC. Food hydrocolloids, 1(1): 65-70. DOI: 10.1016/S0268-005X(86)80008-X

10. Levigne S., Thomas M., Ralet M. C., Quemener B., \& Thibault J. F. (2002), Determination of the degrees of methylation and acetylation of pectins using a C18 column and internal standards. Food Hydrocolloids, 16(6), pp. 547-550.

11. Bondar A. G. (1976), Planirovanie eksperimenta v himicheskoy tehnologii (osnovnyie polozheniya, primeryi i zadachi), Vischa shkola, Kyiv.

12. Mironov V. A., Yankovskiy S.A. (1985), Spektroskopiya v organicheskoy himii. Sbornik zadach. Himiya, Moscow.

13. Braun D., Floyd A., Seynzberi M. (1992), Spektroskopiya organicheskih veschestv. Mir, Moscow.

14. Namazi H., Fathi F., \& Dadkhah A. (2011), Hydrophobically modified starch using long-chain fatty acids for preparation of nanosized starch particles. Scientia Iranica, 18(3), pp. 439-445.

15. Luk’yanenko I. G., KrasnIkova L. I. (1998), Ekonometrika. Znannya, Kyiv.

16. Harris D. C. (2010), Quantitative chemical analysis. Macmillan, New York. 